\title{
Die letzten Tage der letzten Tage der Menschheit in der Fackel
}

Les derniers des derniers jours de l'humanité dans Le Flambeau

The last days of the last days of mankind as presented in The Torch

\section{Gerald Stieg}

\section{(2) OpenEdition}

Journals

Édition électronique

URL : http://journals.openedition.org/austriaca/331

DOI : 10.4000/austriaca.331

ISSN : 2729-0603

Éditeur

Presses universitaires de Rouen et du Havre

Édition imprimée

Date de publication : 1 décembre 2018

Pagination : 61-74

ISBN : 979-10-240-1354-1

ISSN : 0396-4590

\section{Référence électronique}

Gerald Stieg, „Die letzten Tage der letzten Tage der Menschheit in der Fackel“, Austriaca [Online], 87 |

2018, Online erschienen am: 01 März 2020, abgerufen am 28 Januar 2021. URL: http://

journals.openedition.org/austriaca/331 ; DOI: https://doi.org/10.4000/austriaca.331 
Gerald STIEG

Professeur émérite, université Sorbonne Nouvelle-Paris 3

\section{Die letzten Tage der letzten Tage der Menschheit in der Fackel}

Von Anfang an war Karl Kraus von der Aura eines Propheten der Apokalypse umgeben. Selbst der Theologe Hans Urs von Balthasar SJ sah ihn in der Apokalypse der deutschen Seele (1939) unter diesem Zeichen: „Einer aber sitzt, ein böser Magier, in der Stille und sammelt in einem verhaltenen Hass, jahrzehntelang, Zug um Zug, Zeugnis auf Zeugnis, für den letzten Prozess der Kultur: Karl Kraus. [...] Das Weltgeschwür Presse [ist] furchtbar und dämonisch 'objektiv' geworden und mordet die Seele.“ - „Das Haus der Kultur ist abgebrannt und all ihr Gut von innen her fraglich geworden." Der Richter im apokalyptischen Prozess verfügt über eine „blitzende Fechtkunst“, die sich „jüdischer Rabulistik“ verdankt und selbst dem Gericht „unterstellt" bleibt. ${ }^{1}$ Die Analogien Karl Kraus - Apokalypse, bzw. Karl Kraus - Sphäre des Rechts durchziehen die Wirkungsgeschichte der Fackel von Trakl, Loos und Kokoschka über Benjamin, Adorno und Canetti bis Luca Ronconi. Ohne diese apokalyptisch-biblische Färbung beschreibt Brecht die „Methode“ Kraus”:

Mit besonderer Kunst wendet er die Methode kommentarlosen Zitierens an. Oft ist es nur ein Titel, über die unveränderte Wiedergabe einer Rede oder eines Gedichts oder einer Zeitungsnotiz gesetzt, der alles dem furchtbarsten Zweifel preisgibt. Kein Wort hilft diesen Schreibern oder Rednern über die wahrhaft tödliche Stille hinweg, die ihren Auslassungen folgt, ohne Urteil werden sie abgeführt. Eine leere Stelle auf dem Papier lyncht sie. Sie haben sich um den Hals geredet, und man hat sie nicht unterbrochen. Diese Methode ist von allen Methoden dieses Schriftstellers die am wenigsten nachahmbare. Sie setzt voraus den Aufbau eines Raumes, in dem alles zum Gerichtsvorgang wird. Der sie anwendet, muss einzig durch seine große Autorität instand gesetzt sein, sein Schweigen zu einem Urteil zu machen. ${ }^{2}$

1. Hans Urs von Balthasar, Die Apokalypse der deutschen Seele. Bd. III: Vergöttlichung des Todes, Freiburg, Johannes, 1998, S. 49-50 und 63. Ursprünglich erschienen bei Pustet, Salzburg-Leipzig, 1939.

2. Zitiert nach Karl Kraus, Die letzten Tage der Menschheit. Materialien und Kommentare, hrsg. 
Man kann die letzten „Kriegsfackeln“ unter beiden Perspektiven lesen. Kraus beherrscht in der Tat beide Register vollkommen. Es ist aber auch möglich, sie als Kommentar zu den chronologisch ablaufenden historischen Ereignissen zu lesen, als subjektiven Kommentar (gewissermaßen als „Tagebuch“) Kraus’ zur Innen-und Außenpolitik, $\mathrm{zu}$ den Kriegsereignissen und zu den Friedensbemühungen, gleichsam als Rückführung der Kommentare auf ihre historischen und journalistischen Quellen, kurz als Versuche einer Rekontextualisierung. Denn dem Leser der Fackel von 1918 waren Namen und Ereignisse gegenwärtig, die heute längst aus dem allgemeinen Gedächtnis geschwunden sind. Diese Form der Rekonstruktion wäre konträr zu Kraus' eigener Intention. Denn in seinem Selbstverständnis gilt: in hundert Jahren sei es überflüssig, die historischen Details zu kennen, der Kontext der Fackel habe längst aus dem historischen „Einzelfall“ unverwesliche Paradigmen gemacht. Mehr noch: Die Fackel verwandelt eine Zeitungsnotiz in ein literarisches, ethisches, ja religiöses Urteil, Die Fackel ist „Weltgericht“, sie berichtet über „Die Sintflut", sie hält einen „Nachruf“ auf das abgebrannte Haus der Kultur, sie handelt von den letzten Tagen und einer letzten Nacht, sie ist Apokalypse, Enthüllung der Wahrheit. Kraus hat im Grunde Recht behalten, die „Kriegsfackeln“ und Die letzten Tage der Menschheit sind heute lesbar, ohne dass man gezwungen ist, unbedingt nützliche Instrumente wie kolossal montiert von Agnes Pistorius heranzuziehen ${ }^{3}$. Doch im Rahmen einer Tagung über den Untergang von Kakanien kann eine zumindest minimale Kenntnis der historischen Fakten und Akteure dem Verständnis nicht schaden.

Am 15. Oktober 1918 erschien die umfangreichste Kriegsfackel (die fünfzehnfache Nummer 484-498, 240 Seiten), gefolgt von der Doppelnummer 499/500 am 20. November, also bereits nach dem Waffenstillstand. Doch alle Beiträge sind vor dem 3. November verfasst worden. ${ }^{4}$ Die Nummer 501-507 vom 25. Jänner 1919 enthält einen einzigen absatzlosen Beitrag von 120 Seiten, den Nachruf, ein „Requiem“, in dem das "de mortuis nil nisi bene“ in sein Gegenteil verkehrt wurde. Der „wichtigste" Tote ist die k.u.k. Monarchie, deren Agonie sich in den letzten Kriegsfackeln spiegelte und der Kraus keine Träne nachweint.

Die Oktobernummer hatte die übliche Struktur der Kriegsfackel,

von Kurt Krolop, Berlin, Volk und Welt, 1978, S. 281.

3. Agnes Pistorius, „kolossal montiert. “Ein Lexikon zu Karl Kraus „Die letzten Tage der Menschheit", Wien, Ibera, 2011.

4. Im Text zitiert als F 484 bzw. 499 + Seitenangabe. 
ein Gemenge aus Aufsätzen, Glossen, Gedichten, Nekrologen, Notizen über Kraus' rege Vorlesungstätigkeit: von März bis zum 17. Oktober 1918 las Kraus vier Mal in Berlin und zehn Mal in Wien. Im November folgten noch Lesungen am 10., 11. und 24. in Wien. Die Programme dieser Lesungen sind ebenso aufschlussreich wie die Komposition der Fackel-Hefte, die man zum Teil auch als Laboratorium der Letzten Tage der Menschheit ansehen kann. In der vorletzten Nummer hat die Zensur nur mehr an zwei winzigen Stellen eingegriffen: das Zitat „Herzlichen Gruß an Zita. Wilhelm" aus einem Telegramm und ein Satzteil aus der Glosse „Die wichtigsten Fragen der Monarchie im Fluss“, nämlich „Die Verwendung (in Zeiten der Piave und Marne) dieser fatalen Redensart..." Gestrichen wurden die beiden „fatalen“ Flüsse. Der Zensurakt unterstreicht das für Kraus aufschlussreiche Phänomen, dass eine abgestorbene Redensart wieder ihren ursprünglichen Sinn gewinnt, ironisch formuliert durch die Aktualisierung einer anderen Redensart: „Ob wir bald aus dem Wasser sein werden, hat der Artikel nicht verraten." In der Glosse „Von der Blattfront" wurde „Herzlichen Gruß an Zita. Wilhelm“ gestrichen. Die Zensur war äußerst empfindlich, wenn es um kaiserliche Namen ging. Vor dem Hintergrund der Sixtus-Affäre hat dieses authentische vertrauliche Grußwort dem Zensor als offene Ironie, ja Lüge erscheinen müssen. Kraus hat alle zensurierten Stellen, die in die Liste der verbotenen Interpellationen im Reichsrat gehörten, in der Fackel von April 1919 nach dem Protokoll des Reichsrats wiedergegeben, in dem die parlamentarischen Anfragen der Sozialdemokraten (darunter Seitz, Renner und Glöckel) betreffend die zensurierten Stellen in der Fackel enthalten waren. Darunter befand sich auch die kurze Erzählung Der Krieg und das lettische Mädchen von Ferdinand Kürnberger aus dem Jahre 1870 (!), in der der sogenannte Heldentod und das ihn verklärende Vokabular einer Destruktion unterzogen wurden, die in nichts Kraus' Kriegsfackeln nachsteht. Im Allgemeinen kann man aber nur über die Toleranz der kakanischen Zensur staunen, darum sind die wenigen Zensurakte besonders aufschlussreich.

Aus der Fülle des nahezu unüberschaubaren Materials werde ich im Folgenden vor allem einige größere Aufsätze und die Auszüge aus den entstehenden Letzten Tagen der Menschheit analysieren: einen kurzen Abschnitt aus „Die letzte Nacht“ (F 484, 116-125) und das „Lied des Alldeutschen“ (F 499,6-12, mit einem Vorspann, datiert auf den 29. Oktober 1918). Dazu kommt eine Fotomontage (Wilhelm II. in Ritterrüstung) in Fackel 499-500, Vorspann. Die zahlreichen Glossen können nur sporadisch herangezogen werden. Programmatische Bedeutung 
haben die Texte, mit denen die letzten beiden Nummern eröffnet werden: „Ausgebaut und vertieft“ (F 484, 1-12) und „Weltgericht“ (F 499, 1-5). Beide betreffen das Verhältnis Österreichs zu Deutschland, also die Konsequenzen des von Kraus verfluchten "Zweibunds" und der daraus resultierenden "Nibelungentreue“, der "Schulter an Schulter" bis zum „letzten Hauch von Ross und Wagen“ kämpfenden Armeen. ${ }^{5}$ In der Kriegsschuldfrage hat es für Kraus nie einen Zweifel gegeben: der apokalyptische Reiter war von Anfang an der deutsche Kaiser Wilhelm II. Es ist auffallend und sehr aufschlussreich, dass die letzten Zensurakte "Glossen“ betrafen, in denen Wilhelm II. Objekt der Satire war. Besonders interessant ist der Fall der Glosse „An einem Tag“, die nur aus Presseberichten vom 8. Juni 1918 bestand, in denen Auszüge aus Reden des österreichischen und des deutschen Kaisers zur Frage des Friedens parallel wiedergegeben wurden. Während Karl I. in Budapest erklärte, dass er es „für seine Pflicht halten werde, die erste Gelegenheit zu benützen, die sich zur Schließung eines ehrenhaften Friedens bieten wird“, sagte Wilhelm II. vor einem Infanterieregiment, dass die „Entscheidung mit Gottes Hilfe“ fallen werde: „Wenn der Moment eintritt, dann werdet ihr dem deutschen Volk die Stellung erworben haben, die ihm gebührt. Der Friede wird durch euch diktiert und vorgeschrieben werden." (F 474, 140). ${ }^{6}$ Die sozialdemokratischen Abgeordneten konnten sich bei ihrer Anfrage nicht des ironischen Kommentars enthalten, dass diese Zensur eher von einer reichsdeutschen Behörde als von einer österreichischen verfügt worden sei. Symptomatischer Weise blieb die unmittelbar folgende Glosse „An einem Friedenstag“ (F 474, 141) unzensiert, obwohl in ihr im selben Geist Reden Karls I. und Wilhelms II. gegenübergestellt waren, allerdings ohne Nennung der Namen! In dieselbe Richtung ging der Vorwurf gegen den öffentlichen Vortrag des Essays „Das technoromantische Abenteuer“ und des noch nicht gedruckten Textes „Für Lammasch“, in dem der Angriff auf die Neue Freie Presse und deren großdeutschen Paradehistoriker Friedjung, der die Linie der Nibelungentreue verfocht, zensuriert worden war. Kraus - ein seltener Fall identifizierte sich in diesem Text völlig mit der Position des Politikers Heinrich Lammasch. Ein anonymer Brief - ein Musterbeispiel einer fake news - hatte Kraus sogar beim Kriegsministerium als Hochverräter

5. Zu diesen „Redensarten“ siehe Werner Welzig (Hrsg.), Wörterbuch der Redensarten zu der von Karl Kraus 1899-1936 herausgegebenen Zeitschrift „Die Fackel“, Wien, Verlag der Österreichischen Akademie der Wissenschaften, 1999.

6. In F 470 ist die Seite 140 leer; der Text ist wiedergegeben in F 508, S. 70-71. 
denunziert. Ironie der Geschichte: der Akt, mit dem die Vorwürfe gegen Kraus endgültig entkräftet wurden, trug die Unterschrift Lammaschs am 31. Oktober, dem „Tag der Nationalversammlung“, wie Kraus ironisch anmerkte (F 508, 104). Es versteht sich von selbst, dass Kraus ähnlich wie Lammasch einen Separatfrieden und die Position Wilsons verteidigte und mit Hohn die Kehrtwende des „publizistischen Ungeheuers, dessen Feder die Prokura des Blutschachers geführt hat" (i. e. Moriz Benedikt) und in dessen Brust „zwei Drecksseelen“ wohnten, kommentierte, das aus dem verteufelten Wilson, der über Millionen „Verderben, Krankheit und Hunger" gebracht habe, in letzter Minute den Bekenner eines „fast biblischen Apostolats“ (F 499, 15: „Österreichs Fürsprech bei Wilson") für das Völkerrecht zu machen fähig war.

Zum Verständnis des intensiven intra- und intertextuellen satirischen Geflechts sind einige Fakten festzuhalten: Kraus war ein entschiedener Gegner des Krieges von Anfang an. (In „Wir zwei“ , F 640-648, Januar 1924, 1-6, benützt er den Bericht eines Vertrauten Franz Josephs I., um eine satirische Parallele zwischen sich und dem Kaiser zu ziehen: beide als Gegner des Krieges und Propheten des unausweichlichen Ruins). Er hielt das Bündnis zwischen Österreich und Deutschland für verheerend und begrüßte jede Friedensinitiative, darunter das österreichische Angebot eines Separatfriedens, durch das Kaiser Karl I. in den Verdacht des Verrats und der Lüge geriet. Die Frage der diplomatischen Lüge (der an Berlin orientierte Außenminister Czernin hatte behauptet, das Friedensangebot sei von Clemenceau und nicht von Österreich (Sixtus-Brief) ausgegangen) ist ein wichtiges Thema der allerletzten Kriegsfackeln. In diesen Punkten vertrat Lammasch eine Kraus vergleichbare Haltung, die in schroffstem Gegensatz zum Engagement der Neuen Freien Presse und ihres Chefredakteurs Moriz Benedikt stand, in dem Kraus einen Hauptverantwortlichen für den Krieg sah. Wie in den Letzten Tagen der Menschheit verliert er seinen Namen und wird zu einer absoluten Autorität, zu einem ER, zum Herrn der Hyänen, zur Inkarnation des diabolischen Prinzips „Im Anfang war die Presse, und dann erschien die Welt" und "Ich bin des Worts Redaktor, das an dem Ende steht“. Was einen bei der Lektüre dieser Fackeln, ja der Kriegsfackeln in ihrer Gesamtheit, heute am stärksten berührt, ist das Phänomen der „alternativen Wirklichkeit" (sprich Lüge) einerseits und der ungeheuerliche Abstand zwischen der mittelalterlichen Phraseologie und Imagologie (der Kaiser im Harnisch zieht das Schwert) und der Realität des technologischen Krieges (Gas, Flugzeuge, „große“ Kano- 
nen), in dem der Soldat zum "Menschenmaterial“ erniedrigt wurde. ${ }^{7}$ Kraus' Methode der internen Kritik hat als entscheidende richterliche Instanz die Sprache. Das „Weltgericht“ der Fackel ist ein Sprachgericht, eine unerbittliche Demontage der Phraseologie.

\section{Ausgebaut und Vertieft}

Die vorletzte Kriegsfackel beginnt mit der Analyse einer ununterbrochen ad nauseam wiederholten Phrase, nämlich: das Bündnis zwischen Österreich und Deutschland sei „ausgebaut und vertieft" worden. (Die Kritik an dieser Wortkoppelung taucht bemerkenswerter Weise erst 1918 in der Fackel auf, also in einem Moment, in dem dieses Bündnis nicht zuletzt aufgrund der Sixtus-Affäre mehr als fragwürdig geworden war.) Die linguistisch-juridische Dekonstruktion der Phrase durch die Methode des selbstzerstörerischen Zitats, in der Kraus virtuos seine unerhörte vis comica entfaltet, führt zu einer überraschenden Schlussfolgerung. Auf die Anhäufung der leerlaufenden Versicherungen über das unverbrüchliche Bündnis, das unentwegt ausgebaut und vertieft, von IHM (Moriz Benedikt) „ausgelegt“ und zu dem biblischen Gebot "Ausgebaut und vertieft sollst Du werden!“ erhoben wird, lässt Kraus einen Bericht aus einer Schweizer Zeitung vom 30. Mai 1918 folgen, in dem die „Militärkonvention zwischen China und Japan“ aufgelistet ist, die der völligen Unterwerfung Chinas durch Japan entspricht. Sein Kommentar: „Mit einem Wort - das Bündnis zwischen Japan (sprich Deutschland) und China (sprich Österreich) ist ausgebaut und vertieft."

Nach dem Krieg hat er die Koppelung der beiden Verben und der entsprechenden Substantive Ausbau und Vertiefung auf völlig andere Bereiche übertragen und sogar die Variante "ausgebaut und verflacht" ironisch verwendet. Das Wörterbuch der Redensarten in der „Fackel“ von Werner Welzig enthält einen eigenen, sehr umfangreichen Eintrag "ausgebaut und vertieft", d. h. die Geschichte einer Phrase, die sich selbständig gemacht hat. ${ }^{8}$ Im Kontext von Ende 1918 ist die Phrase nicht nur Phrase, sondern Musterbeispiel dessen, was als „nackte Fälschung

7. Vgl. dazu Peter Sloterdijk, Luftbeben. An den Quellen des Terrors, Frankfurt am Main, Suhrkamp, 2002. Nach Sloterdijk war Kraus einer der ersten, der das terroristische Potenzial des Einsatzes von Giftgas diagnostiziert hatte.

8. Werner Welzig, op. cit., S. 123-137. 
der Tatsachen" (F 484, 14) bezeichnet werden kann. Kraus handhabt virtuos die dialektische Umkehrung der Phrase in einer diplomatischen Verwicklung von höchster Bedeutung. Sie wird zu einem Lieblingsmuster nichtssagender Phraseologie auf allen Ebenen von ihrem politischen Ursprung über eine andere zentrale Phrase (die „Hebung des Fremdenverkehrs") bis zu kulturellen Phänomenen (z. B. Burgtheater). Hin und wieder geschieht es in politischen Reden, dass die Sprache die Wahrheit verrät, wenn das , ausgebaute und vertiefte Bündnis“ mit den Metaphern des „Joch" und der „Ankettung" umschrieben wird. Besonders interessant ist eine Stelle in einer Rede des vorletzten kaiserlichen deutschen Außenministers Paul von Hintze über das „Bündnis“ vor der Wiener Presse. Nachdem er erklärt hatte, dass die Bündnispartner "durch Opfer, Leiden und Triumphe unauflöslich aneinander gekettet" seien, definierte er das Bündnis so:

Unser Bündnis ist ein wirkliches „Bündnis“. Ich gebrauche ausdrücklich das Wort „Bündnis“ ohne jeden Zusatz. Irgendein adjektivistisches Beiwort würde den Begriff nur abschwächen können (F 484, 37).

Das Gespenst eines „Scheinbündnisses“ ist allerdings durch das Adjektiv ,wirklich“ mitbeschworen. Kraus kann sich nicht der Frage enthalten, ob nicht unter Hintzes Hörern „leidenschaftliche Anhänger adjektivistischer Beiwörter" waren - eine Spitze gegen den feuilletonistischen Stil à la Stefan Zweig. In unzähligen Varianten hat Kraus die Brüchigkeit und Nichtigkeit dieses Bundes persifliert, nicht zuletzt in seinem Porträt Franz Josephs I. Die pointierteste Form hat seine Kritik daran im Nachruf erhalten:

Und das muss man ja sagen: wenn je in der Tragödie missleiteter Völker ein weltgeschichtlicher Humor mitgespielt hat, so wurde er von dem Anblick dieses in die Kriegsmaschine geratenen Charakterbreis bestritten, der, angekettet an eine Kapazität der Dressur die fremde Tonlage - (auch „von dieser unerbittlichen Melodie der Treue gequält“ -) durchhalten musste, in seiner angeborenen Stimmung zwischen „Wer' mr scho machen“ und „Kann man halt nix machen“ an der Seite eines machenden und schaffenden Ungeheuers kläglich verzappelt ist und wirklich eher den feindlichen Angriffen in die Front als den fortwährenden Freundesstössen in die Weichteile gewachsen war (F 501, 70).

Der Wahrheit ganz nahe kommt folgende Aussage des Außenministers Burián (F 484, 38): „Das 'deutsche Joch' ist für Österreich-Ungarn 
das Joch der beiderseitigen felsenfesten Freundschaft und vollen Rücksichtnahme auf die Interessen beider Teile [...]“. Dieser Generalbass, gebildet aus „Ausbau und Vertiefung“, erlaubt spezifische Varianten, deren prägnanteste ohne jeden Zweifel die Affäre um die sogenannten "Sixtus-Briefe“ ist. Denn wie sonst nichts dementiert das geheime Friedensangebot Kaiser Karls I. das „wirkliche Bündnis“ und die „felsenfeste Freundschaft". Handelt es sich bei der ständigen Beschwörung der Bündnistreue um eine Verschleierung des wirklichen Verhältnisses, so geht es in der Sixtus-Affäre um offene Vorwürfe der Lüge. Von den Lügnern wird nach Kraus die Wahrheit als „,nackte Fälschung der Tatsachen“ bezeichnet. Zugespitzt formuliert: „Wenn die Lüge ein Kriegsmittel ist, so ist jene Lüge, deren Inhalt die Behauptung ist, dass der andere gelogen hat, ein Dum-Dum-Geschoss" (F 484,14).

\section{Berichtigung}

Kraus hat sich ein grimmiges Vergnügen daraus gemacht, Zeitungen durch „Berichtigungen" nach dem $\$ 19$ des Pressgesetzes einer doppelten Rechtsprechung auszusetzen: dem Gesetz des Staates und dem Gesetz der Fackel. Solche Berichtigungen konnten anscheinende Kleinigkeiten betreffen, z. B. den berühmten Streit um eine falsche Interpunktion oder die Richtigstellung von Daten, z. B. des richtigen Erscheinungsdatums der Fackel. Benjamin, Canetti und viele andere haben den gesetzgebenden und richterlichen Charakter der Fackel hervorgehoben. Und es scheint mir in der Tat sinnvoll, die Fackel als einen einzigartigen Ort der „Berichtigung“ im Geiste des eingangs zitierten Brechtzitats zu lesen. Berichtigt werden Falschmeldungen und Lügen. Die Form, die diese Berichtigung annehmen kann, ist äußerst variabel. Schon quantitativ betrachtet, reicht sie von winzigen, kommentarlosen Zitaten (wenn man vom Titel absieht), bis zu riesigen Essays wie "Nachruf“ oder „Warum die Fackel nicht erscheint“ (F 890 bis 905, 1933). Aus dem Kontext der letzten Kriegsfackeln isoliere ich zwei Beispiele der „nackten Fälschung von Tatsachen", das erste betrifft die bereits erwähnte Sixtus-Affäre, das zweite die Frage der Ernährung im Krieg.

Eine einzige knappe Glosse mit dem Titel „In Erwartung“ (F 484, 21) illustriert perfekt die Methode. Es handelt sich offensichtlich um einen Auszug aus der Neuen Freien Presse: 
Über die Behauptungen Clemenceaus, betreffend die Briefe des Kaisers Karl, wird auf die heutigen offiziellen Veröffentlichungen hingewiesen, durch welche diese Briefe als absolut falsch und erfunden bezeichnet werden. Clemenceau gibt ja auch keine weiteren Einzelheiten über diese Briefe und erwähnt gar nicht, was sonst in diesen Briefen enthalten sein soll. Doch können wir in Ruhe weitere Enthüllungen abwarten. Eine etwaige Wiedergabe von Facsimiles wird sich zweifellos als Schwindel, als Fälschung herausstellen. Denn dass diese Briefe Falsifikate sein müssen, steht heute absolut fest.

Diese undatierte Zeitungsnotiz stand in der Fackel vom 15. Oktober und hatte sich seit der Veröffentlichung der Briefe durch Clemenceau selbst erledigt. Am 2. April 1918 hatte Außenminister Czernin behauptet, das Ansuchen um Verhandlungen für einen Separatfrieden sei von Clemenceau ausgegangen. Clemenceau „antwortete“ mit der Veröffentlichung des ersten Sixtusbriefes, in dem der Kaiser Frankreich u. a. die Wiedergewinnung von Elsass-Lothringen anbot. Der Kaiser, der dementiert hatte, war als Lügner und gleichzeitig als Verräter an der "Nibelungentreue“ stigmatisiert. Der Generalstabschef Arz sagte: „Ich habe erfahren, dass mein Kaiser lügt." Diesem durch die Tatsachen dementierten „In Erwartung“ war eine Serie von Glossen vorangegangen, in denen Kraus mit „rabulistischem“ Witz die verzweifelten Versuche Czernins und der ihm ergebenen Presse, die Lüge den Franzosen zuzuschieben und zu versichern, „In unserer äußeren Politik steuern wir Gott sei Dank den deutschen Kurs“ (F 484, 13), kommentiert.

\section{Weltgericht}

Die letzten Kriegsfackeln enthalten auch Literaturpolemiken, allen voran die Auseinandersetzung mit Franz Werfel in „Das Ich und das Ichbin“. Der Zusammenhang mit der Kriegsthematik ist auch hier, allerdings nur am Rande, spürbar. Kraus denunziert die Autoren, die im Kriegspressequartier arbeiten, aber auch die Kriegskritiker wie Zweig oder Rolland, die dies gefahrlos in der Schweiz, also im neutralen Ausland, tun. Zwei Totengedenken für die gefallenen Freunde Franz Grüner und Franz Janowitz rahmen in der vorletzten Nummer einen Auszug aus der Letzten Nacht, die letzte wird eröffnet mit einer „Deutschen Postkarte“ „Unser Kaiser in Harnisch!“. Sie trägt die Unterschrift Wilhelms II., den Slogan „In Treue und in Waffen fest“ (+ eisernes Kreuz) 
und Sätze aus einer Rede des Kaisers: „Wir Deutsche fürchten Gott und sonst absolut nichts und niemanden auf dieser Welt." Dieses Foto ist im Grunde ein konzentriertes Symbol für den Anachronismus der Ritterrüstung im "chlorreichen“ Gaskrieg. „Das Lied des Alldeutschen“ versammelt das gesamte Repertoire an Phrasen und Floskeln der Ideologie des m.w. (machen wir!), also des "machenden und schaffenden Ungeheuers", als das Kraus das wilhelminische Deutschland identifizierte. Die beiden Fragmente gehören in den Prozess des anbrechenden „Weltgerichts“. Die nach Kraus treibenden Mächte des Kriegs sind in diesen Szenen aus den Letzten Tagen der Menschheit mythologisch überhöht: die liberal-kapitalistische Presse, inkarniert im „Herrn der Hyänen“, der deutsche Imperialismus im „alldeutschen“ Kommerzienrat Ottomar Wilhelm Wahnschaffe der Letzten Tage, der in der Fackel noch namenlos ist. Dieses von ihm selbst komponierte Lied gehörte bereits zum Repertoire der Lesungen Kraus' während des Krieges und wurde in Die letzten Tage der Menschheit übernommen. In der Fackel folgt ihm das Gedicht über den österreichischen Bündnispartner unter dem Titel „Mir san ja eh die reinen Lamperln“ (F 499, 13-14). Kraus hat das „Lied des Alldeutschen“ mehrfach selbst kommentiert, zuletzt in „Warum die Fackel nicht erscheint“: der Nationalsozialismus erscheint dort als perfekte Verwirklichung der Ideologie Wahnschaffes. Es gibt auch eine groteske Seite der Wirkungsgeschichte des Liedes, eine Illustration dessen, was Kraus als ,verfolgende Unschuld“ definiert hat. Der Kritiker Alfred Kerr, der während des Krieges scheußliche Kriegslyrik rassistischer Tendenz produziert hatte, versuchte allen Ernstes Kraus der Verunglimpfung und Verleumdung der deutschen Nation zu überführen. Das 21 neunzeilige Strophen lange Gedicht - eine Schwäche der satirischen Lyrik Kraus' ist ihr Drang zur Vollständigkeit, sprich ihr Katalogcharakter - versammelt alle Elemente der Kritik, die Kraus in der Fackel gegen das wilhelminische Deutschland vorgebracht hat. Er gehörte noch zu jenen Intellektuellen, die zwischen ihrem guten Deutschland (Goethe und Kant, ja sogar Bismarck) und dem „,neudeutschen" Ungeheuer aus Romantik und Technik unterschieden haben. Ich zitiere hier nur die rekurrenten sprachlichen Elemente, die in das Gedicht einmontiert sind:

[...] immer feste druff, Heil Krupp und Krieg, die Wacht am Rhein, Platz an der Sonne, ich frage nicht nach Gut und Geld, das Lebensmittel ist uns Zweck, Kunst im Dienste des Kaufmanns, Walhalla, fromm und frei, von der Hand in den Mund leben, Gold gab ich für Eisen, deutscher Gott, Fenris-Wolffbüro, 
Ersatz, Wille zur Macht, Machen wir (um Zeit zu sparen, auch: m.w.), und wenn die Welt voll Teufel wär, Fibel, viel Feind viel Ehr, Deutschland über alles, Gott strafe England, der deutsche Gott, gaudeamus igitur, muntrer Bursch, Volk der Denker, Bildung-schmücke dein Heim, mit Gott für Krupp und Vaterland, durchhalten, Siegfriedstellung, mit Bomben belegt, Gottes Ebenbild ist nur der Deutsche, Annexion, Belgien geben wir nicht her, Ehrenkleid, Endsieg, made in Germany, Gift und Gas, es braust ein Ruf wie Donnerhall, Ausbau der Technik, U-Boot, Fortschritt, Und wenn die Welt voll Teufel wär.

Von Frundsberg, der in den Letzten Tagen zum „Bilanzknecht“ geworden ist, über die Burschenschafter-Romantik und die Nationalisierung des Psalms „Ein feste Burg“ bis zur imperialistischen Macher-Ideologie, die mit Hilfe des deutschen Gottes einen Siegfrieden erstreiten will, sind alle Themen der Kriegsfackel auf engstem Raum versammelt. In den Letzten Tagen wird dieses „Porträt“ Deutschlands ergänzt durch die Beschreibung der Villa Wahnschaffe (Butzenscheiben und Schrebergartenromantik + Kriegspropaganda) und die Familien - und Ernährungsideologie von Gattin und Kindern Wahnschaffe (III, 40).

Der Auszug aus der Letzten Nacht ist kommentarlos, Kraus resümiert bloß die vorangegangenen und nachfolgenden Szenen. Unmittelbar vor der Szene hatte Ing. Siegfried Abendrot den Verrat der Wissenschaft an die chemische und biologische Kriegsführung gerühmt. Kraus entwirft die unheimlichen Konsequenzen einer durchaus denkbaren biologischen Kriegsführung mittels der Lungenpest, an der die deutsche Forschung konkret arbeitete. In der Namengebung (Dr. Ing. Siegfried Abendrot) vereinen sich Technologie, Kriegsziel (Siegfrieden) und germanische Mythologie (Tod durch die Nibelungenpest).

Ganz anderer Natur ist die Episode der „Hyänen, die Menschengesichter tragen“, vor allem aber die sprechenden Namen „Fressack“ und "Naschkatz“. Auf einen Nenner gebracht repräsentieren die Hyänen das Kapital, das die Fusion zwischen Bank und Schlachtbank zustande gebracht hat. Sie sprechen zu den toten Soldaten, deren Heldengrab ihnen "Schab“ (Gewinn) gebracht hat. Ihre Söhne sind dem Kriegsdienst entkommen, u. a. dadurch, dass sie Kriegsgedichte schrieben und dafür im Kriegspressequartier Unterschlupf gefunden haben. Kurz: die Hyänen sind die Inkarnation der Kriegsgewinner. Über ihnen waltet der riesenhafte „Herr der Hyänen“, schon aufgrund der Beschreibung seiner Bart- und Haartracht als Moriz Benedikt zu erkennen. Sein Monolog in 22 Strophen ist ein radikal anti-christliches und antihumanistisches Bekenntnis, das mit Bibelzitaten gespickt ist. ER macht den 
gekreuzigten Christus vergessen: „Ich tret' an seine Stelle: / die Hölle ist die Helle!: Ich bin der Antichrist.“ „Er wollt die Welt erlösen; / sie ist von ihm erlöst." An Stelle der christlichen Werte, vor allem des Liebesgebots, treten das Geld („das große Ringen mit vielen Silberlingen“), die Macht („Die Macht nur ist der Wert!“), Rache und Hass. An Stelle des Reiches Gottes ist die Herrschaft des Teufels angekommen: „Ich bin sein erster Faktor (Drucker!), / ich bin des Worts Redaktor, / das an dem Ende steht“. Sein „Reich ist von dieser Welt“. Zwei Szenen der Letzten Tage der Menschheit, die Gegenüberstellung von Papst Benedikt xv. und Moriz Benedikt (I, 27 und 28), werden so umformuliert: „Der alte Pakt zerreiße! / So wahr ich Moriz heiße, / der Wurf ist uns geglückt! / Weil jener andre Hirte / sich ganz gewaltig irrte! / Ich heiße Benedikt.“ Der „andere Papst“ hat dank der „schwarzen Magie“ triumphiert: „Durch die geheime Finte / zum Treubund rief die Tinte / die Technik und den Tod.“ Die Schlussfolgerung: „Ich traf mit Druckerschwärze / den Erzfeind in das Herze! / Und weil es ihm geschah, / sollt ihr den Nächsten hassen, / um Judaslohn verlassen - / der Antichrist ist da!“ Dieser Auszug aus der kommenden Tragödie arbeitet nicht mehr mit der von Brecht beschriebenen Technik, sondern ist ein Musterbeispiel von bibelgesättigter Intertextualität.

\section{Die Lebensmittelmächte (F 484, 205)}

Dieses Wortspiel ist vielschichtig. Zunächst beruht es auf einer Grundlinie von Kraus’ Denken, nämlich, dass in der modernen Gesellschaft das Lebensmittel zum Lebenszweck geworden sei. Doch in der Glosse „Unzeitgemäße Redensarten“ verbindet es Kraus mit der Versicherung des ungarischen Handelsministers, „dass das Bündnis der Völker längst zu Fleisch und Blut geworden sei. Sie ist immer und zumal im vorliegenden Falle deplaziert: was das Fleisch betrifft, als Lüge, und was das Blut betrifft, als Wahrheit.“ Er empfiehlt den Staatsmännern und Publizisten der Lebensmittelmächte ihre Sprache, die noch zu viele Viktualien enthält, zu revidieren. Denn „es ist schwer, durchzuhalten, wenn man bei jedem Brocken der Sprache an jeden Brocken erinnert wird.“ „Fleisch, Blut, Früchte des Waldes (Brombeeren), Viktualien, Brocken (sc. Brot)“ gehören zu einem Wortfeld der großen Zeit: das Blutbündnis führt dazu, dass es kein Fleisch und Brot mehr gibt, aber Kraus geht von der Hypothese aus, dass man im Krieg „fleischlose Wochen leich- 
ter als phrasenfreie Tage erträgt“. Das „Publikum“ isst sich an Phrasen satt. Eine solche Phrase ist neben „ausgebaut und vertieft“ für Kraus auch der „Brotfriede“ (d. h. der Friede von Brest-Litowsk): laut österreichischen und deutschen Regierungsverlautbarungen sind die Getreidevorräte in der Ukraine viel größer als die Transportkapazitäten der Lebensmittelmächte. Kommentar Kraus':

So triumphiert über die Erkenntnis, dass es kein Getreide aus der Ukraine gibt, immerhin der Trost, dass es Getreide in der Ukraine gibt, und das Durchhalten wird zum Kinderspiel, wenn einen der sichere Brotfriede sowohl für das Brot wie für den Frieden entschädigt (F 484, 24-25).

Die Nahrungsfrage bewegt sich nicht immer auf diesem metaphorischen Niveau, sie ist brutale Wirklichkeit. In den Letzten Tagen der Menschheit steht dafür die emblematische Szene (IV, 18): die Kinder rufen: „Vater, Brot!“ Der Vater antwortet: „Kinder, Russland verhungert!“

In der Fackel findet sich unter der Überschrift „Lügen der Entente“ (F 484, 181) kommentarlos der Zeitungsartikel „Lynchjustiz an einer Kartoffelkäuferin“:

Wien, 28. Juni (1918). In Stammersdorf bei Wien wurde gestern eine bisher unbekannte Frau, die dort Kartoffeln gekauft hatte, von anderen, aus Wien gekommenen Personen, die nichts mehr bekommen hatten, überfallen und erschlagen $[\ldots]$

Darauf folgt die Glosse „Ausbau und Vertiefung“: sie beginnt mit einem Zitat über die „Monarchenbegegnung“ (Karls I. und Wilhelms II.), die natürlich zu Ausbau und Vertiefung geführt hat. In der Zeitungs-Kolumne steht das in unmittelbarer Nachbarschaft zu: „Neuerliche Verschlechterung der Wohnungsverhältnisse in Wien“, „Neuerliche Erhöhung der Milchpreise in Wien“ und „Herabsetzung der Brotration in Deutschland von Mitte Juni an." Kraus kommentiert abschließend: „Ob Ausbau Herabsetzung und also Vertiefung Erhöhung bedeutet, ließ sich in dem Chaos nicht unterscheiden."

Die Brot- und Ernährungsfrage hat noch eine andere, die medizinisch-wissenschaftliche Seite. Die Glosse „Es ist alles da, es ist nicht so wie bei arme Leute“ (F 484, 180), die in unmittelbarer Nähe der Glossen über den tödlichen Kampf um Kartoffel und Brot steht, ist besonders aufschlussreich. Sie beginnt mit einem langen Zitat aus einer Agentur- 
meldung des Wolff-Büros. Kraus hat dieses Organ der deutschen Propaganda immer wieder in Anspielung an die germanische Mythologie als Fenris-Wolff-Büro bezeichnet, das die Schuld am Weltbrand trägt. In der zitierten Meldung werden Nachrichten englischer Zeitungen „über den angeblich schlechten Ernährungszustand der deutschen Bevölkerung“ dementiert. Der „Gegenbeweis“ wird durch Umfragen unter 6000 deutschen Krankenkassen, vor allem aber durch die Aussagen des „Ärzteausschusses für Groß-Berlin“, geliefert. Er läuft darauf hinaus, dass im Krieg dank der gesunden Ernährung viele Krankheiten und die Sterblichkeit im Rückgang seien. „Ärztlicherseits [wurde] ausdrücklich die Bekömmlichkeit der gegenwärtigen Kriegskost festgestellt." Kraus kommentiert: „Ach, wenn es doch immer so bliebe! Oder: Das war eine herrliche Zeit!“ Er spielt auch auf den „Schöpfer des 'Obu'“, der Bratpfanne „ohne Butter“ an. Wie weit die Erfindungskraft im Bereich der kriegsbedingten Kochkunst und Nahrungsmittelchemie gehen konnte, illustriert die Glosse „Das deutsche Wunder, der Neid der Welt“ (F 484, 64-65), deren Kern der folgende Triumph des „reinen Geistes über die rohe Materie“ ist: Professor Delbrück ist es gelungen, „Mineralnährhefe" aus Harnstoff zu erzeugen. Er hofft, in Zukunft sogar als rohe Materie „Harn und Jauche heranzuziehen.“

Der „Ersatz“ ist ein großes Thema der Letzten Tage der Menschheit. Die Glosse „Das deutsche Wunder, der Neid der Welt“, ein wichtiger Beitrag zur Thematik der „Nachrichten [...], die allesamt mit den Tatsachen in direktem Widerspruch stehen“, gestattet auch einen kleinen Einblick in die Werkstatt der Tragödie. Professor Delbrück hat in ihr eine eigene Szene: III, 10. Sie ist ein Konzentrat aus der Glosse, doch Kraus behielt es sich vor, die ursprünglichen Zitate zu arrangieren und z. B. Delbrück nicht nur seine eigenen Worte, sondern auch die des Ärzte-Ausschusses für Groß-Berlin in jenen Mund zu legen, der Nahrungschemie im Dienste des Krieges propagierte. In der Frage der Authentizität der Zitate im Drama könnte man Kraus (nicht nur an dieser Stelle) der Manipulation zeihen, in der Fackel regiert jedoch unangefochten der Geist und die Form des „Zeugnisses“ vor dem „Weltgericht“. 